\title{
Author Correction: Band gap closure, incommensurability and molecular dissociation of dense chlorine
}

\author{
Philip Dalladay-Simpson (10) 1, Jack Binns (1) 1, Miriam Peña-Alvarez (D) 2, Mary-Ellen Donnelly (1) 1, \\ Eran Greenberg (1) ${ }^{3}$, Vitali Prakapenka ${ }^{3}$, Xiao-Jia Chen (1) ${ }^{1}$, Eugene Gregoryanz (1) ${ }^{1,2}$ \& Ross T. Howie (1) ${ }^{1}$
}

Correction to: Nature Communications https://doi.org/10.1038/s41467-019-09108-x, published online 08 March 2019.

The original version of this Article omitted references to previous experimental reports on solid hydrogen that are relevant for a full understanding of the context of the previous work. The added references are:

47. Akahama, Y. et al. Evidence from x-ray diffraction of orientational ordering in phase III of solid hydrogen at pressures up to 183 GPa. Phys. Rev. B 82, 060101 (2010).

48. Zha, C.-S., Liu, Z. \& Hemley, R. J. Synchrotron infrared measurements of dense hydrogen to 360 GPa. Phys. Rev. Lett. 108, 146402 (2012).

49. Dias, R. \& Silvera, I. Observation of the Wigner-Huntington transition to metallic hydrogen. Science 355, 715-718 (2017).

50. Eremets, M. I. \& Drozdov, A. P. Comments on the claimed observation of the Wigner-Huntington transition to metallic hydrogen. Preprint at http://arxiv.org/abs/1702.05125 (2017).

51. Loubeyre, P., Occelli, F. \& Dumas, P. Comment on: "Observation of the Wigner-Huntington transition to metallic hydrogen". Preprint at http://arxiv.org/abs/1702.07192 (2017).

52. Goncharov, A. F. \& Struzhkin, V. V. Comment on "Observation of the Wigner-Huntington transition to metallic hydrogen". Science 357, eaam9736 (2017).

53. Liu, X.-D., Dalladay-Simpson, P., Howie, R. T., Li, B. \& Gregoryanz, E. Comment on "Observation of the Wigner-Huntington transition to metallic hydrogen". Science 357, eaan2671 (2017).

Citations to these reference, plus reference 21, have been added to the fourth sentence of the Introduction: 'The experimental realisation of atomic metallic hydrogen has remained elusive despite intense research efforts lasting over 30 years ${ }^{4-7,21,47-53}$.' This has been corrected in the PDF and HTML versions of the Article.

\footnotetext{
${ }^{1}$ Center for High Pressure Science Technology Advanced Research, 1690 Cailun Road, Shanghai 201203, China. ${ }^{2}$ Centre for Science at Extreme Conditions, School of Physics and Astronomy, University of Edinburgh, Edinburgh EH9 3FD, UK. ${ }^{3}$ Center for Advanced Radiation Sources, University of Chicago, Chicago, IL 60637, USA. Correspondence and requests for materials should be addressed to P.D-S. (email: philip.dalladay-simpson@hpstar.ac.cn) or to R.T.H. (email: ross.howie@hpstar.ac.cn)
} 
(c) Open Access This article is licensed under a Creative Commons Attribution 4.0 International License, which permits use, sharing, adaptation, distribution and reproduction in any medium or format, as long as you give appropriate credit to the original author(s) and the source, provide a link to the Creative Commons license, and indicate if changes were made. The images or other third party material in this article are included in the article's Creative Commons license, unless indicated otherwise in a credit line to the material. If material is not included in the article's Creative Commons license and your intended use is not permitted by statutory regulation or exceeds the permitted use, you will need to obtain permission directly from the copyright holder. To view a copy of this license, visit http://creativecommons.org/licenses/by/4.0/.

(C) The Author(s) 2019 